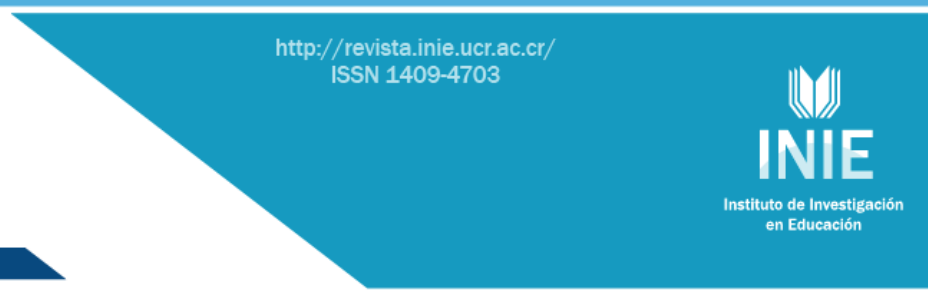

\title{
INTERCULTURALIDAD: JUEGO Y METODOLOGÍAS DE AULA DE PRIMARIA
}

MULTICULTURALISM: GAME AND METHODOLOGIES IN THE PRIMARY SCHOOL CLASSROOM

\section{Volumen 16, Número 1}

Setiembre-Diciembre

pp. $1-19$

Este número se publicó el $1^{\circ}$ de setiembre de 2016

DOI: http://dx.doi.org/10.15517/aie.v16i3.26067

Maurizia D' Antoni

Revista indizada en REDALYC, SCIELO

Revista distribuida en las bases de datos:

LATINDEX, DOAJ, E-REVIST@S, IRESIE, CLASE, DIALNET, SHERPA/ROMEO, QUALIS,

Revista registrada en los directorios:

ULRICH'S, REDIE, RINACE, OEI, MAESTROTECA, PREAL, CLACSO 


\title{
INTERCULTURALIDAD: JUEGO Y METODOLOGÍAS DE AULA DE PRIMARIA MULTICULTURALISM: GAME AND METHODOLOGIES IN THE PRIMARY SCHOOL CLASSROOM
}

\begin{abstract}
Maurizia D’Antoni ${ }^{1}$
Resumen: El ensayo, en un contexto de experiencia investigativa de la autora donde emerge la enorme exposición de niños y niñas a los videojuegos, pretende explorar la importancia de la parte afectiva y emocional en la niñez y, en lo específico, el papel que recubre el juego en el desarrollo, la exploración de la realidad y construcción de sí a través de la actividad lúdica, el juego de roles y el papel de los juegos electrónicos en la actualidad. El origen y el rol del juego son discutidos en un plano filogenético, como parte del desarrollo de la persona, y se evidencia su relación con el plano cultural; mientras que la temática de la interculturalidad aparece ligada a la diferencia cultural (no solo en el juego) y a la importancia de las culturas en las aulas.

Palabras clave: ESTRATEGIAS EDUCATIVAS, JUEGO Y ESCOLARIDAD, DESARROLLO INFANTIL, INTERCULTURALIDAD.

Abstract: The essay, in the context of the research experience of the author attesting the enormous current exposure of children to video games, it explores the importance of affective and emotional side in childhood and specifically the role that the game represents in development, exploration and construction of reality itself, through activities, role play and the role of electronic games for the current childhood. The origin and role of the game are discussed on a phylogenetic level, as part of the development of the person, but the implications of the game in the cultural sphere are also taken into account; while the theme interculturalism appears linked to cultural difference (not just about the game) and the importance of cultures in the classroom.
\end{abstract}

Key words: EDUCATIONAL STRATEGIES, GAME AND SCHOOL, INTERCULTURALISM, CHILD DEVELOPMENT

\footnotetext{
1 Profesora e investigadora catedrática de la Universidad de Costa Rica, donde labora en la Escuela de Psicología y en el Departamento de Docencia Universitaria. Es catedrática e investigadora en la Universidad Nacional, División de Educología, donde es parte del proyecto Alfabetización crítica en la cultura escolar.
}

Dirección electrónica: maurizia.dantoni@gmail.com

Ensayo recibido: 14 de octubre, 2015

Enviado a corrección: 12 de mayo, 2016

Aprobado: 22 de Agosto, 2016 


\section{Introducción: El papel del juego en la época escolar}

La intención de este artículo es explorar la importancia del desarrollo afectivo y emocional en la niñez, al vincularlo con el papel del juego. En otras palabras, me interesa investigar la construcción de la realidad y la construcción de sí en la niñez, a través de la actividad lúdica. Así mismo, el artículo indagará acerca del juego de roles y sobre el origen del juego en un plano filogenético. Este análisis toma en cuenta el desarrollo de los niños y las niñas en el plano individual, pero sin desvincularlo del contexto cultural y su lugar en él.

Para iniciar, considero relevante tomar en cuenta la contribución del psicólogo ruso Lev Vygotski, haciendo referencia a su trabajo en general y a como lo interpreta Wanda Rodríguez en cuanto puede aclarar el papel del juego o la actividad lúdica en los niños y las niñas en edad preescolar y escolar, pues ha sido reconocido como un estudioso de la cognición y, en ese sentido, es posible que otras lecturas de su obra no le hayan dado el espacio que merece (Rodríguez, 2011). No obstante, además de las temáticas asociadas a la cognición, este autor ruso se interesó siempre en el tema de la afectividad y las emociones ligadas a la temática del juego. Así, pues, para entender su obra en su entereza es importante incorporar la dimensión emocional y afectiva para la comprensión del sujeto cognitivo.

Ahora bien, el cambio de paradigma dentro de la comunidad científica especialista llamó, en su momento, "revolución cognitiva" al proceso que trató de trascender el sujeto reactivo conductista al permitir el desarrollo de una concepción de sujeto como activo manipulador de signos. Luego, Vygotski realizó una segunda revolución para impulsar una visión del sujeto capaz de transformar y construir significados y sentido (Rodríguez, 2011). Adicionalmente, el enfoque histórico-cultural del trabajo de este autor se ha encargado de reconocer e incorporar en sus desarrollos teóricos y en la investigación "el lugar de las emociones y la afectividad en las explicaciones de las acciones humanas" (Rodríguez, 2011, p.30). Este reconocimiento se ha dado de manera cautelosa para que la temática no se degrade como sucedió en los casos de otras contribuciones como, por ejemplo, con el concepto de "zona de desarrollo próximo". ${ }^{2}$

\footnotetext{
2 El concepto de Zona de Desarrollo Próximo en Vygotski, el decir la distancia de lo que el niño o la niña puede realizar en su desarrollo consolidado y lo que puede construir con la ayuda de un adulto o de un par más, ha sido interpretado y discutido de manera contrastante (Baquero, 2009). Inclusive se señala (Rodríguez, 2014) una probable deformación de sus intenciones en cierta literatura que quiso identificar una especie de estadio del desarrollo.
} 
Con respecto al tema de la actividad lúdica, Vygotski (2000) considera inadecuado estimar al placer como característica definitoria y principal del juego. Asimismo, reconoce la importancia del este como actividad ligada al desarrollo, pero lamenta que al describirlo se tomen en cuenta solamente las funciones intelectuales. Su propuesta, entonces, pasa por redefinir el juego ubicando el tema del placer como uno más, no el fundante, entre otros atributos. Esta concepción radica, según el autor, en que existen juegos donde lo que se realiza no es placentero en sí mismo, el placer viene con la conclusión, por ejemplo, con el hecho de ganar un juego.

La percepción humana se completa, en los años tempranos, a partir de la capacidad de descubrir objetos reales, pero este descubrimiento no solo tiene que ver con la forma o el color de los objetos, sino con la apreciación de su significado. Este se elabora a partir de generalizaciones en un contexto de clasificación de los objetos, más que de discernimiento de características aisladas. De igual forma, las características de estos objetos también tienen su significado. Esto quiere decir que, en el panorama más general del juego, no cualquier pieza puede convertirse en un elemento específico del juego mismo. Vygotski (2000) ejemplificaba lo anterior argumentando que un niño o niña puede usar un palo para representar un caballo, pero nunca transformará una postal en un caballo.

Durante el juego, el niño opera con significados separados de sus objetos y acciones acostumbradas; sin embargo surge una interesante contradicción en que funda las acciones reales y los objetos reales. Esto caracteriza la naturaleza transicional del juego; es un estadio entre las limitaciones puramente situacionales de la temprana infancia y el pensamiento adulto, que puede estar totalmente libre de situaciones reales. (Vygotski, 2000, p.150-151)

En la situación del palo que, al cabalgarlo, se convierte en caballo, el objeto influye semánticamente en el otro con una transferencia de significado, donde una palabra es aceptada como la propiedad de un elemento, pero lo que se ve no es la palabra, se ve más bien lo que esta palabra señala. Así, pues, en la infancia, se actúa primero a través de un significado y no a través del objeto (teniendo en cuenta el ejemplo del palo que es caballo). Luego, estos actos se llevan a cabo conscientemente y el teórico advierte, en este punto, que llega un momento cuando el o la infante sabe los detalles pero no advierte que los sabe. Esto sucede en una época previa a la adquisición de la lectoescritura, cuando se separa el objeto de su significado. 
Es interesante la reflexión de Vygotski (2000) acerca de la autorregulación del niño o la niña durante el juego. Como se mencionó más arriba, este no se constituye siempre en una actividad placentera y para llegar a una gratificación final, puede ser que la persona tenga que vivir situaciones que en otro contexto le resultarían difíciles o que no soportaría si no mediara la actividad lúdica. En esta, el impulso inmediato se aplaza, pues las reglas del juego a veces se oponen a lo que la persona joven quisiera hacer en ese momento. Vygotski dice, de manera sapiente que: "el mayor autocontrol del que es capaz un niño se produce en el juego. Alcanza el mayor despliegue de poder cuando renuncia a una atracción inmediata en el juego" (p.151). Así pues, las reglas internas construidas por el niño o la niña en su juego brindan:

(...) una nueva forma de deseos. Le enseña a desear relacionando sus deseos con un "yo" ficticio, a su papel en el juego y sus reglas. De este modo, se realizan en el juego los mayores logros del niño, logros que mañana se convertirán en su nivel básico de acción real y moralidad. (Vygotski, 2000, p.152)

Aquí, el autor vincula también el juego con la construcción de reglas morales, esto es desde un pensamiento moral entendido como una sofisticación cognitiva la cual permite que la persona pueda concertar posturas en la moralidad corriente o bien, pueda interpretarla críticamente.

En nuestras observaciones en las aulas, las cuales se llevaron a cabo en el contexto de las prácticas realizadas en la Escuela de Psicología de la Universidad de Costa Rica, o en el curso de otras investigaciones (D' Antoni, 2009; Aguiar y D' Antoni, 2008), una de las indicaciones hechas por el profesorado tuvo que ver con las dificultades de los niños varones, sobre todo para permanecer sentados en sus sillas por largo tiempo, en un espejo de aula de tipo tradicional con filas de sillas y la docente al frente. Otro señalamiento involucró las dificultades que tienen para mantener la atención y concentración por parte de sus estudiantes, igualmente realizando una única actividad por periodos sostenidos de tiempo.

Justamente para apoyar una actividad formativa en el aula que se sirva de la motivación, de elementos afectivamente cargados en pro del aprendizaje del cuerpo estudiantil, es útil una reflexión sobre las actividades del tiempo libre de niños y niñas. Su conocimiento puede ser aprovechado si se leen estas desde un enfoque histórico cultural que las enmarque. 


\section{Juego y videojuegos}

Los juegos electrónicos representan una modalidad interesante de juego infantil que merece ser enfocada en el contexto del interés por lecturas histórico-culturales de la actividad de niños y niñas. Se trata de especulaciones que pueden conseguir indicaciones de rumbo actuales para la actividad formativa. Efectivamente, una de las actividades con más adeptos y que más interesa a los estudiantes hombres, un poco menos a las estudiantes mujeres, son los videojuegos. Cuando se indaga acerca de los pasatiempos desarrollados en el tiempo libre o sobre los héroes o juguetes favoritos, este tipo se encuentra entre las preferidas de los escolares costarricenses.

Pasar el tiempo frente a un videojuego, en efecto, es una actividad que requiere sostener la atención y visualizar secuencias repetidas y patrones iguales, una y otra vez, realizando operaciones mentales de cierta complejidad, como prever el lugar a donde disparar o cómo evitar un obstáculo. De la misma manera, están involucradas la coordinación oculo-motora, así como la habilidad motora fina para el manejo del comando que transfiere el impulso a la pantalla, por dar un ejemplo.

Con respecto a esta modalidad en la cual hay tecnologías aplicadas al juego, y tomando en cuenta la perspectiva de Vygotski (1978, 2000), los videojuegos no retribuyen a los niños y las niñas de manera inmediata; por el contrario, suministran a menudo frustración, como cuando la persona que juega pierde ante la máquina. A la vez, aun perdiendo, el deseo es avanzar de nivel y enfrentar modalidades de juego siempre más complejas y donde dicha complejidad se acrecienta. Así las cosas, con una aparente contradicción; estudiantes de escuela primaria, aparentemente incapaces de permanecer inmóviles en sus pupitres por largo tiempo, pueden, en cambio, realizar la hazaña de la inmovilidad en un sillón de la casa o de una sala de videojuegos, al frente de una pantalla. Claramente la contradicción aparente tendría que ser estudiada: la hipótesis que se puede asomar es que esto se debe a que en la escuela no se juega, que a menudo en el salón de clases falta la emoción, la participación, o el compromiso con la cultura del niño o de la niña

Considerando los aportes de Vygotski (2000), nos damos cuenta de que "el atributo esencial del juego es una regla que se ha convertido en deseo" (p. 152) y nos preguntamos, entonces, si no será posible involucrar al estudiantado en un nuevo deseo en el aula, transformando las actividades curriculares en juegos ( $y$, por qué no, videojuegos), interrogando a los pequeños y las pequeñas acerca de sus ídolos, de sus culturas y sus saberes. Dicho esto, en lugar de sucumbir a la fascinación de las máquinas o de la 
electrónica por sí sola, las tecnologías podrían convertirse en un gancho creador de motivación para el estudio. En este marco, los cursos de robótica insertados en algunos planteamientos curriculares actuales podrían tener la función indicada, es decir, se aprende y utiliza la matemática en un sentido aplicado y, de esta forma, los conocimientos son directamente involucrados en la creación, construcción y gestión de un juego en movimiento. Cabe señalar que los cursos de robótica vinculados únicamente a la faceta técnica no obtendrían los mismos beneficios, cuando el enfoque fuera el que se critica, es decir el que toma la técnica como un valor en sí mismo, a prescindir de un enfoque capaz de leer el entorno histórico y cultural.

Si la escuela primaria pública tiene alguna relación con lo que sucede en la secundaria, se ha visto que (D’Antoni, Gómez, Gómez y Soto, 2013) uno de los problemas que enfrentan las dinámicas actuales en el aula de primaria en Costa Rica es la falta de atracción para niños y niñas. De hecho, este factor podría considerarse dentro de las razones del abandono escolar, el fracaso y la repitencia. El juego, en cambio, tiene el factor de la motivación, de la capacidad de desarrollar concentración, autocontrol y, en el caso del videojuego, la capacidad de autoinstruirse a través de los crecientes niveles de complejidad. A la vez, diferentes tipos de juego están involucrados directamente con el desarrollo y el aprendizaje, niveles que, para Vygotski, se influencian mutuamente (Vygotski, 2000).

Ahora bien, el tema del autocontrol en las aulas involucra también la capacidad de empoderar al niño o a la niña y alfabetizarlos críticamente (D’ Antoni, Gómez, Gómez y Soto, 2013), esto para que ellos, de alguna manera, encuentren no solamente actitudes de resistencia, sino respuestas posibles ante la expulsión educativa y el fracaso escolar masivo (Baquero, 2009). En este contexto, el psicólogo educativo Ricardo Baquero insiste en la necesidad de que se desarrollen "las condiciones que generen prácticas educativas apropiadas y apropiables" (Baquero, 2009, p.21). En este panorama, el autor reitera que las aulas son los lugares aptos para el aprendizaje, a la vez que discute la creencia de que el fracaso escolar masivo sea percibido como la suma de fracasos individuales.

Utilizando el juego como metodología para el trabajo en el aula y, al mismo tiempo, recuperando las culturas y los gustos de las personas jóvenes, se motiva el aprendizaje y se permite, asimismo, un trabajo a favor de la alfabetización crítica y del empoderamiento de la población juvenil. Si el enfoque pedagógico decide hacerse cargo del mencionado fracaso escolar masivo, lo cual parece ser una realidad en varios países de América Latina y ciertamente en Costa Rica (Programa Estado de La Nación, 2011, 2013), el trabajo formativo 
deberá trabajar en pro de una alianza entre docentes y estudiantado para mantener a este último grupo en las aulas y conscientizarlo de los procesos expulsivos que enfrenta en el sistema educativo.

En este esquema, enfatizo la importancia de la capacidad de autorregularse, es decir, de adaptarse de manera consciente y pragmática al contexto educativo, pues es clave para no enfrentar procesos de exclusión o expulsión activa dentro de un sistema homogeneizante que presenta los contenidos en modo fragmentario y que potencialmente atenta contra los procesos de desarrollo cognitivo más de lo que contribuye a apuntalarlos (D' Antoni. Gómez, Gómez y Soto, 2013).

\section{Juego, proceso indispensable}

En este panorama, los niños y las niñas se emancipan de las limitaciones situacionales mediante la "situación imaginaria". Sobre este tema específico de la "situación imaginaria" en el juego ronda un gran interés y ha sido estudiado hasta la actualidad, como se cita a continuación.

En el campo particular de la educación, el juego infantil fue considerado, en un principio, como una actividad natural que lleva en sí enormes posibilidades de aprendizaje y desarrollo (Ortega, 1991). La necesidad de cambiar esquemas tradicionales que vinculaban el aprendizaje con la transmisión de ideas o habilidades para dominarlas posteriormente, así como la visión de situaciones cotidianas en un plano de desarrollo espontáneo por parte del niño o la niña, nos indica hoy que el juego es, en cambio, un escenario privilegiado para analizar el desarrollo (Ortega, 1991).

Ahora bien; por una parte, a través del tema del juego, Vygotski (2000) explora la proporción existente entre objeto/significado y acción/significado en la niñez. En la edad preescolar, por ejemplo, es la acción la que predomina sobre el significado, el cual puede ser entendido solo parcialmente. Por otra parte, la niña o el niño no actúan de manera puramente simbólica en el juego, existe un deseo que se realiza a través de este y en este la experiencia media, las categorías básicas de la realidad. El autor pone el ejemplo de unos infantes que juegan a comer. En este caso, las acciones, aún en niños y niñas preescolares, siguen reglas y una estructura que imita a la realidad: el plato, los brazos hacia delante y no hacia atrás, la acción de fingir el uso de herramientas (cubiertos o elementos de cocina), entre otros. 
En cuanto a la relación acción/significado: golpear el suelo fingiendo ir a caballo o manipular una manopla imaginaria como si se estuviera dando gas tienen la valencia de una inversión significado/acción. Existe una especie de trampolín entre la acción real que no se puede realizar y la que se realiza. La acción aparece en un segundo plano y su significado se separa de la acción mediante otra acción diferente.

En este recorrido, vislumbramos el camino hacia la adquisición de la simbolización y la ubicación de la persona infante entre la comprensión y la adquisición de esta y de sus características.

El operar con el significado de las cosas nos lleva al pensamiento abstracto, el desarrollo de la voluntad y la capacidad de llevar a cabo elecciones conscientes se producen cuando el pequeño opera con el significado de la acciones. En el juego una acción sustituye a la otra, igual que un objeto reemplaza al otro. (Vygotski, 2000, p.183)

Se aprecia un movimiento en el campo del significado típico del juego, este representa el movimiento en un campo abstracto, con un método situacional y concreto. Luego, el proceso se completará con la operación de incorporar lo simbólico como signo, y se llegará al intercambio de estos. Notamos aquí la aparición del campo del significado, donde la acción desarrollada tiene características iguales a las de la realidad.

El juego para Vygotski es mucho más que una de las actividades típicas de la infancia, es un proceso indispensable para el desarrollo de los niños y las niñas, donde la construcción de la situación imaginaria migra a la construcción del juego, con sus reglas.

Hemos visto cómo las reglas son un elemento básico del juego más "avanzado", y en lugar de ser algo que aumenta la frustración en aquel que desarrolla la actividad, se convierte en un elemento de placer, de construcción de la autorregulación y del auto conocimiento infantil. Ahora bien, el plano de la construcción de la regla en el juego infantil puede ser un elemento de provecho en muchos ámbitos educativos. Aquí podemos subrayar su papel en la didáctica y vincularlo además con el juego de roles, pues esta modalidad tiene implicaciones relevantes y aprovechables en el plano educativo.

\section{Juego, aula, interculturalidad}

Proponemos aquí un seguimiento de la metodología de enseñanza empleada: talleres que intentaban responder inquietudes del profesorado al enfrentar la diversidad cultural en las aulas (Aguilar y D' Antoni, 2009) y el tema del juego en la infancia. La preocupación que 
mueve a proponer tal temática es que en la actualidad, la metodología en el contexto escolar no comprendan actividades retadoras que llamen la atención de los niños y las niñas y que, además, apoyen el desarrollo (D’ Antoni, Gómez, Gómez y Soto, 2013).

Después de haber discutido lo que Vygotski (año) plantea acerca del juego, interesa ahora identificar el uso del concepto: a pesar de que en la literatura especializada no existe una definición consensual sobre qué es el "juego", se ha tratado de identificar características que lo distinguen de otras formas de actividad o comportamiento humano, por ejemplo:

a) una actividad no literal en la que la realidad es leída de manera diferente, asignando a objetos, personas y acciones, otros significados, sociales y propios;

b) una característica de flexibilidad con ideas y comportamientos novedosos utilizados en la situación lúdica;

c) prevalencia de medios sobre fines con una actividad;

d) el tema del placer y la diversión en los y jugadoras, en un contexto afectivamente positivo;

e) se trata de una actividad voluntaria, libremente seleccionada;

f) el control: los jugadores controlan y tienen un nivel de determinación (nivel: ver, por ejemplo, el caso de los videojuegos) del curso del desarrollo de los eventos;

g) el juego es una actividad en situación, es decir, depende del contexto o el lugar donde se realiza;

h) finalmente, se trata de una actividad resolutoria de problemas y situaciones que los niños y las niñas plantean y que en condiciones comunes no podrían hacer por sí solos (Dalton, 1996).

Ahora bien, entre las expresiones del juego existe uno sociodramático: aquel en el cual el niño adopta roles sociales conocidos y en el que utiliza objetos para representar diferentes acciones. Como se ha mencionado, la teoría de Lev S. Vygotsky $(1978,2000)$ le reconoce un rol central al juego en el desarrollo de los procesos de pensamiento y en la alfabetización, así como al desarrollo integral que involucra emociones y cognición. En lo específico, las representaciones durante el juego simbólico hacen que el niño o la niña aprenda que un objeto puede estar en el lugar de otro (simbolización) y use estas representaciones simbólicas "de primer orden" en sus esfuerzos para el aprendizaje de la lectoescritura. Así, la escritura y el juego en el pensamiento vygotskiano aparecen como momentos diferentes en el proceso de transformación que conduce hasta el desarrollo del lenguaje escrito. 
La investigación clásica de Mergen (1991) reflexiona sobre la dificultad de encontrar la diferencia entre los juegos y las actividades lúdicas que la niñez prefiere, y los que las personas adultas (profesorado, supervisores de juegos etc.) piensan que niños y niñas deben practicar. Aunque el estudio analiza las preferencias de juego en la ciudad de Washington, Estados Unidos, ofrece, a su vez, un recorrido histórico sobre las preferencias de juegos y sobre las modificaciones de esas preferencias a través de la historia (Mergen, 1991).

Por su parte, Ignacio Dalton (1996) y Dalton y Christie (1998) han propuesto la metodología de los "centro lúdicos". En este esquema, niños y niñas se comprometen a realizar un diseño de ambiente de juego con su docente, a través de un proceso de concreción de micro tareas en las cuales construyen, diseñan y desarrollan actividades para el funcionamiento de los centros. Es una estrategia que se ha denominado "diseño cooperativo de los centros lúdicos" (Dalton, 1996) y que puede proporcionar directrices sobre la utilización del juego en los contextos del aula.

\section{El juego en la interculturalidad}

En el contexto del aula de primaria costarricense, donde en las observaciones encontramos una vez ocho nacionalidades diferentes (D' Antoni, 2009), una de las preguntas que pueden plantearse al pensar en la actividad lúdica infantil es: ¿son los juegos iguales en todas las culturas? Esta incógnita sugiere un desplazamiento del tema del desarrollo infantil de un nivel individual a uno más macro; en otras palabras, sugiere un descentramiento del desarrollo infantil en el sujeto, para dar cuenta del desarrollo infantil de los sujetos sociales dentro de un marco cultural. En este ámbito, es legítimo preguntarse si el juego tiene la capacidad de expresar las diferencias culturales y si estas sirven, entre otras cosas, para reconstruir las rutas de la socialización y de la aculturación o bien, para acompañar a los niños y a las niñas en su proceso educativo y de adaptación cultural.

Janot (2011) es uno de los autores que se inclina por privilegiar una visión unitaria e intercultural del juego. Ello analiza considerando la dimensión espacial, la cual se caracteriza como uno de los elementos determinantes de su lógica. Un estudio taxonómico y comparativo entorno al juego motor tradicional evidencia concordancias y disparidades que emergen de la comparativa de cinco catálogos de juegos localizados en zonas geográficamente distintas. El propósito de su investigación es juzgar si el patrimonio lúdico humano evidencia, más allá de su multiculturalidad o de un enfoque intercultural, una 
substancia transcultural. Así, pues, su investigación toma en cuenta prácticas de juego descritas en catálogos que se realizan en países tan diversos como Perú, República Dominicana, España o Marruecos. El estudio compara y responde preguntas sobre las concordancias y las disparidades que los catálogos de juegos de una determinada área geográfica mantienen con respecto a otros populares y tradicionales que se hayan desarrollado a lo largo del tiempo en otros espacios geográficos.

La investigación evidencia una relación entre espacio y manejo de materiales en el juego, pues, así como el espacio sirve para fijar el desafío entre adversarios o instigar la confrontación entre jugadores, el tiempo funciona de la misma manera. De igual forma, la investigación concluye que el cierre del juego, motivado por la realización de una tarea determinada, es un aspecto que los catálogos comparten.

A partir de la comparación de los catálogos lúdicos, el estudio propone la existencia de un isomorfismo lúdico al afirmar la presencia de varias analogías en el accionar y las dinámicas del juego. No obstante, esa homogeneidad disminuye cuando las categorías de espacio, tiempo y material entran en el análisis. Finalmente, la investigación invita a abrir nuevos caminos que se focalicen en una búsqueda, más concluyente, de rasgos lúdicos transculturales. Por un lado, la tesis de Janot (2011) sugiere que el juego se desarrolla en una manera similar en los diferentes espacios geográficos, a pesar de que tiene lugar en culturas distintas. Por ejemplo existen juegos, como el de esconderse, o "policías" que cazan "ladrones" que se repiten en diferentes culturas con modalidades diferentes. Por otro lado, el autor se propone ampliar el estudio en futuras investigaciones y tomar en cuenta otras culturas lúdicas; además de estudiar un mayor número de catálogos de juegos con el objetivo de confirmar su hipótesis: el patrimonio lúdico de la humanidad muestra, más allá de su multiculturalidad, rasgos transculturales.

Por su parte, Fidel Molina (2010) investiga el tema de las reformas educativas como producto de los enfoques de las diferentes políticas educativas que piensan los objetivos de la educación en función de las nuevas necesidades sociales, económicas y culturales. Las necesidades en cuestión han sido investigadas mediante un diagnóstico educativo de la realidad sociopolítica. De acuerdo con este autor, dentro del esquema con el que se llevan a cabo las reformas educativas, el profesorado aparece como uno de los agentes clave. Reseña una serie de investigaciones desarrolladas dentro del periodo 2000 y 2010, con efoques cualitativos y cuantitativos, en las que se destaca la voz del profesorado. Uno de los temas centrales y en los que enfatiza tiene que ver con la diversidad cultural. Las reflexiones 
teóricas revisadas por Molina (2010) incluyen experiencias desarrolladas en España; toma en cuenta la transformación educativa en Argentina (básicamente la región NOA), y se destacan estudios sobre el desarrollo de la educación intercultural y la educación intercultural bilingüe en México, Brasil y Guatemala.

La investigación de Molina (2010) se contrapone a la de Janot (2001), aun cuando los dos casos consideran la temática de las diferencias culturales en varios países. Para empezar, el primer teórico privilegia en buena medida el área iberoamericana. En segundo lugar, Janot (2001) evidencia una plataforma común de la cual se desprenden los juegos, independientemente de la cultura base, mientras que los resultados de Molina (2010), en cambio, expresan la intención de buscar orientaciones provechosas para la reflexión y la acción educativa (sociocultural y política) en un contexto donde se evidencia el llamado "drama del desarrollo" causado por los megaproyectos de transformación educativa. De acuerdo con este autor, los megaproyectos promueven la segmentación educativa cuando coexisten varios sistemas educativos en un mismo país. Adicionalmente, sugiere Molina (2010), el modelo integrador de la institución educativa que nace con la modernidad necesaria para que ser repensado y cuestionado.

La investigación de Molina desarrolló un trabajo de campo en el cual se usaron diversas técnicas de trabajo: observación participante, entrevistas y encuestas. A través del implemento de estas técnicas y durante el transcurso de la investigación, el autor estimó que los centros educativos que trabajan con un sentido de la interculturalidad y la inclusión integran la voz del profesorado, el cual, a su vez, se encuentra apoyado por las familias y la administración. En este contexto, señala la relevancia de la buena formación del personal y los directivos; el planteamiento de proyectos claros y el desarrollo de actividades que relacionan el centro educativo con el entorno, así como la presencia de un monitoraje que apoye al profesorado, facilitando la relación interpersonal y la orientación estudiantil.

La escuela de la diversidad aparece entonces como una respuesta de la modernidad tardía, la cual descubre nuevas vinculaciones entre lo universal y lo particular. En este sentido, mientras la escuela tradicional se vincula a través de una dicotomía ficticia, el contexto de pluralismo asegura la igualdad e integración.

De igual forma, Molina (2010) considera que en la escuela se percibe un reflejo de los problemas sociales. En otras palabras, estos son reproducidos en la escuela y, de esta manera, el centro educativo y sus integrantes participan de la exclusión y la discriminación 
que están presentes en los entornos sociales. Así, problemáticas como la discriminación y marginación social recaen sobre la institución escolar.

El enfoque de esta investigación desplaza por completo el interés en el fenómeno juego, el cual parece expresarse de la misma manera en ambientes culturales diversos, a la relación existente entre el funcionamiento interno de la institución educativa, que quiere decirse sensible a los cambios, y a la presencia en su seno de sujetos con proveniencias y /o de formaciones culturales diversas. Se quiere, entonces, vincular el tema de la integración cultural en instituciones educativas al evidenciar los factores necesarios para que esta se dé, a saber: atención a la gerencia institucional, comunicación entre profesorado y con las familias y la existencia de planificación consciente que tome en cuenta una direccionalidad centro-periferias para articular acciones.

De esta manera, más que la sugerencia acerca de sugestiones interculturales a través del juego, que surgiría de manera espontánea y universalista de culturas distantes, el foco central apunta a los actores y actrices del cambio en el nivel institucional y en los procedimientos en la planificación que pueden unir y poner a hablar a poblaciones diversas de forma justa e inclusiva.

Por su parte, el trabajo de González, Solovieva y Quintanar (2009) es dedicado a la actividad de juego temático de roles en la formación del pensamiento reflexivo; se enfoca en preescolares y en parte a niños y niñas de los dos primeros años de la primaria. La investigación mexicana toma en cuenta juego de roles como actividad preparatoria, en el nivel preescolar, de la constitución de neo formaciones que a su vez darán lugar a habilidades básicas para que los pequeños y las pequeñas puedan trabajar provechosamente en la escuela primaria. A la vez, el juego de roles se utiliza como una actividad evaluativa del nivel de desarrollo del cuerpo estudiantil. Su investigación, en efecto, se convierte en material de referencia a la hora de buscar alternativas de acción para prevenir retos en el aprendizaje de escolares desde la etapa preescolar. La intervención de esta perspectiva se propone como innovadora y se realiza por etapas para abordar la formación gradual de la actividad intelectual, en el salón de clase, según las características de la edad psicológica.

La investigación (González, Solovieva y Quintanar, 2009) muestra el recorrido del juego de roles como apoyo para la construcción, con la capacidad de identificación de los objetos del mundo social, de la capacidad simbólica, voluntaria, reflexiva, comunicativa desplegada e imaginativa. En efecto, son imaginación, volición, simbolización, reflexión las 
capacidades que se han asociado en México con índice de bajo desempeño escolar en los primeros años de la primaria (Moreno, Solovieva y Quintanar, 2007); se trata de construcciones mentales que podrían ser importante monitorear en niños y niñas costarricenses, en un contexto de fracaso escolar masivo (Baquero, 2001) en la primaria, debido a que ha sido, justamente, el fracaso masivo en lectoescritura lo que ha llevado al Ministerio de Educación Pública costarricense a trasladar el acostumbrado objetivo de saber leer y escribir del primero a segundo año de escuela primaria (Díaz Rojas, 2013). Esta preocupación acerca del desempeño de escolares de escuela primaria y estudiantes de colegio costarricense se extiende a los hechos relativos a las pruebas diagnósticas de segundo ciclo en 2010, las cuales revelaron que solo el $20 \%$ de los y las estudiantes de sexto grado alcanza las habilidades lingüísticas esperadas para su nivel. Comentando temas relativos a la adquisición de la lectoescritura, además, los alcances obtenidos en Costa Rica en la prueba internacional PISA $^{3}$ señalaron que el $67 \%$ de los jóvenes costarricenses posee una comprensión de lectura poco más allá de lo básico (Díaz Rojas, 2013).

Los investigadores y la investigadora en México (González, Solovieva y Quintanar, 2009) detectaron que, en un inicio, niños y niñas preescolares hacían referencia a los objetos materiales y a su representación para resolver tareas de manera dependiente del adulto o de pares. Paulatinamente, se asistió, en cambio, a una evolución compleja para lograr la forma independiente. En este contexto, los objetos materiales concretos, perceptuales y generalizados utilizados partieron de su naturaleza exterior como instrumentos objetivos; al ser transformados en interiores, se hicieron instrumentos internos y subjetivos, es decir, parte de la relación íntima del niño o de la niña. De esa forma, se puede comprender que el lenguaje de las personas adultas quienes educan es clave en la relación que niños y niñas establecen con los objetos del mundo externo y con los otros, en el contexto de la organización de acciones que tienen un objetivo establecido. Luego, es el infante quien toma la decisión independiente sobre qué escoger y cómo realizar lo que pretende llevar a cabo: con el apoyo de su lenguaje verbal externo, niños y niñas se encaminan a lograr la volición autónoma, entre otras adquisiciones del desarrollo.

Las conclusiones del estudio de González, Solovieva y Quintanar (2009) apuntan a que los niños y las niñas del grupo experimental con el cual trabajaron no contaban con la

\footnotetext{
${ }^{3}$ Las Pruebas PISA (Programme for International Student Assessment) son cuestionados instrumentos de evaluación mundial, que de manera generalizada y con un movimiento desde "centro" hacia una periferia, véase el nombre en inglés por ejemplo, pretenden medir y comparar, entre otros aspectos, la comprensión lectora de jóvenes a nivel mundial (Zapata Ríos, 2015).
} 
preparación y con herramientas para el desarrollo "exitoso" del aprendizaje escolar y, de la misma manera, su nivel para el pensamiento reflexivo era bajo. Adicionalmente, el equipo investigativo cree que el enfoque histórico cultural constituye una aproximación provechosa para la prevención de dificultades en el aprendizaje y para apoyar, de esta manera, un desarrollo psicológico óptimo en la edad preescolar. En este contexto, el juego temático de roles, en las aulas de preescolar, posibilita el desarrollo de formaciones psicológicas que garantizan el mejor aprendizaje en esta edad y alejan el fantasma del fracaso escolar masivo.

El trabajo de González, Solovieva y Quintanar (2009) destaca, a la vez, el papel de la Zona de desarrollo próximo para la evaluación del desempeño preescolar y escolar, ya que, gracias a ella, se pudo establecer el nivel de desarrollo actual y potencial en cada niño o niña, de manera individual, y en los grupos de manera más general. Asimismo, las autoras y el autor consideran que una de las fortalezas de la investigación se dio por el hecho de que el cuerpo docente participante en el proceso enriqueció, a la luz del estudio realizado, sus herramientas de trabajo. De esa forma, se llevaron a cabo acciones en el salón de clases que facilitaron y fortalecieron el desarrollo del estudiantado: el profesorado aprendió a identificar sus necesidades al integrarlas a su quehacer pedagógico; es decir, sensibilizándose frente a sus motivaciones e intereses.

Finalmente, se enfatiza en el hecho de que el artículo, en el sentido intercultural, toma en cuenta la motivación, la cultura y los intereses infantiles y juveniles de los estudiantes en las aulas, para hacer de este acervo el centro desde el cual debe partir la actividad pedagógica. Así al igual que en Vygotski (2000), se asimila que el recorrido inicia desde el apoyo adulto y desde los objetos concretos del mundo material y avanza hacia la interiorización de un mundo preexistente de "cosas" y de significados. Este asume una función socializadora que se posibilita en el desarrollo integral los juguetes y el juego, entre estos, el juego de roles.

En la herencia costarricense, que incluye las reflexiones sobre la práctica educativa, emerge Carmen Lyra, escritora, educadora, política y teórica de la educación. En 1926 y 1927, la autora abogaba por la alegría en las aulas, la libertad de movimiento instintivo o inteligente; lo anterior se conforma en un patrimonio que en la vida adulta se convertiría en optimismo creador (D’ Antoni, 2015). Lyra también divulgó el tema del derecho al movimiento de niños y niñas escolares, de la importancia del recreo como momento integrado de la actividad formativa, de la necesidad de que las escuelas tuvieran juegos y espacio al aire 
libre en dotación, y, explícitamente, de la importancia del juego como elemento por integrar en la práctica educativa. En sus ensayos sobre educación, dedicó espacio a la temática de la construcción de juegos al aire libre como toboganes o carruseles en los patios de las escuelas (D' Antoni, 2015).

Cabe resaltar el enfoque visionario y adelantado a su tiempo de la educadora costarricense, quien, a partir de su experiencia en las aulas, se opone, ya en 1926 y 1927, a la educación tradicional, aburrida, que constriñe al cuerpo infantil, lo encierra y desprecia, por lo tanto, el potencial emocional y motivacional que se alcanzaría si las personas docentes aprovecharan el juego en sus acción formativa.

\section{Conclusiones}

A través de este escrito, se ha querido plantear el tema del papel que recubre el juego en el desarrollo al destacar también la importancia de la parte afectiva y emocional en la niñez, la potencialidad de exploración de la realidad y de construcción de sí a través de la actividad lúdica en niños y niñas. Más que investigar "novedades", este documento ha pretendido vincularnos con la teoría histórica cultural de Vygotski (2000), caracterizada por la convicción que, en un contexto de crecimiento del ser humano como tal en comunidad, aprendizaje y desarrollo son dos caras de la misma moneda. Lo anterior implica una responsabilidad especial del ámbito educativo con un contexto formativo comprometido con la comunidad y las mejoras sociales, reflexivo y creativo.

Acerca del tema de las tecnologías, parece relevante verificar de alguna manera su vigencia a través de la exploración del tema de los videojuegos.

Interrogándose acerca de la interculturalidad, el juego asume un espacio específico en la cultura de cada país y en nuestra actualidad, así que el juego de roles y, una vez más, el papel de los juegos electrónicos muestran cómo en este momento histórico, las educadoras podrían apropiarse conscientemente de actividades que son tan importantes y placenteras, tan cargadas afectivamente para sus escolares, en beneficio de un buen desarrollo integral infantil.

Es necesario preguntarse acerca del origen del juego en un plano filogenético, como elemento del desarrollo de la persona, pero también problematizando este tema como actividad para el aprendizaje a la luz de la pluralidad de culturas que observamos hoy en las aulas costarricenses. 
La investigación de González, Quintanar y Solovieva (2009) evidencia la importancia de aprender a identificar las necesidades del estudiantado por parte del cuerpo docente, de sensibilizarse ante las motivaciones e intereses de niños y niñas: reconocer y desarrollar motivaciones, intereses, culturas de las personas presentes en el aula.

Específicamente la investigación mexicana coincide con Vygotski (2000) al reconocer el papel complejo de la labor docente en la cual la motivación estudiantil se reconoce como una vertiente por trabajar. Los niños y las niñas interiorizan nuevas adquisiciones a la par de un mundo preexistente de "cosas", complementadas por sus significados. En este proceso, la motivación es una de las responsabilidades docentes, empezando por la comprensión de la dinámica que la sostiene. Los juguetes y el juego, y entre estos el juego de roles, son herramientas centrales para cumplir con la función socializadora de la educación.

El desarrollo integral, así conceptualizado, es clave para crecer juntos y juntas en una escuela que estimula y reta, que reconoce e incluye, un tema que ya casi hace cien años proponía la educadora costarricense Carmen Lyra.

\section{Referencias}

Aguiar, Montealegre, Tatiana y D' Antoni, Maurizia. (2008). Taller de mediación cultural para maestros y maestras costarricenses. Revista Reflexiones, 87(1), 113-126. Recuperado de http://revistas.ucr.ac.cr/index.php/reflexiones/article/view/11489/10835

Janot, Jaume, Bantulá. (2011). Espacio e interculturalidad en el juego popular y tradicional. Bienvenidos, 10, 17-38.

Baquero, Ricardo. (2001). La educabilidad bajo sospecha. Cuaderno de Pedagogía, 4(9), 7185. http://www.profesaulosuna.com/data/files/EDUCACION/PSICOLOGIA\%20EDUCATIVA La\%2520educabilidad\%2520bajo\%2520sospecha.pdf

Baquero, Ricardo. (2009). Zona de desarrollo próximo, sujeto y situación, el problema de las unidades de análisis en psicologìa educativa. Actualidades Investigativas en Educación, 9(Número Especial), 1-25. Recuperado de http://revista.inie.ucr.ac.cr/index.php/aie/article/view/307

D’ Antoni, Maurizia. (2009). Vygotski, desarrollo infantil y aportes para el aula costarricense. Actualidades Investigativas en Educación, 9(Número especial), 1-15. Recuperado de http://revista.inie.ucr.ac.cr/index.php/aie/article/view/306

D’Antoni, Maurizia. (2015) Carmen Lyra: una pedagogía acallada en Costa Rica y sus vinculaciones teóricas. II Congreso Internacional de Investigación Educativa. Educación y Globalización. INIE, Universidad de Costa Rica. Recuperado de http://inie.ucr.ac.cr/tercercongreso/memoria/documentos/3/carmenlyraunapedagogiaacallada.pdf 
D’ Antoni, Maurizia, Gómez, Ordoñez, Luís, Gómez, Torres, Juan y Soto Arguedas, José Fabio. (2013). La Escuela en Cuestionamiento. Diálogos sobre la resistencia escolar en procesos pedagógicos emergentes. San José: Arlekin

Dalton, Ignacio, y Christie, James, F. (1998). El juego y sus implicancias educativas en el aprendizaje de la alfabetización. Lectura y Vida, 18(1), 64-71.

Dalton, Ignacio. (julio, 1996). Contribuciones para la alfabetización con niños por medio del juego. Ponencia presentada en el Congreso Internacional de Educación. Facultad de Filosofía y Letras, Departamento de Ciencias de la Educación, Universidad de Buenos Aires.

Díaz, Rojas, Katherine. (18 de Siembre, 2013). Nuevos programas de Ministerio buscan mejorar la enseñanza de la lectoescritura Recuperado de http://www.mep.go.cr/noticias/nuevo-programa-espanol-busca-mejorar-ensenanzalectoescritura

Programa Estado de La Nación. (2011). Tercer Informe Estado de la Educación en Desarrollo Sostenible. San José, Costa Rica: Estado de la Nación.

Programa Estado de La Nación. (2013). Cuarto Informe Estado de la Educación en Desarrollo Sostenible. San José, Costa Rica: Estado de la Nación.

González Moreno, Claudia Xiména, Solovieva, Yulia y Quintanar Rojas, Luís. (2009). La actividad de juego temático de roles en la formación del pensamiento reflexivo en preescolares Revista Internacional de Investigación en Educación, 2(3), 173-190.

Mergen, Bernard. (1991). Ninety-five years of historical change in the game preferences of American children. Play \& Culture, 4(3), 272-283.

Molina, Fidel. (2010). La interculturalidad y el papel del profesorado en las reformas educativas. Ra Ximhai, Universidad Autónoma Indígena de México Mochicahui, Sinaloa, 6(1), 131-143.

Moreno, Claudia, Quintanar, Luís y Solovieva, Yulia. (2007). Neuropsicología y aprendizaje escolar. Revista Ciencia y Desarrollo 33(2), 287-308.

Ortega, Rosario. (1991). Un marco conceptual para la interpretación psicológica del juego infantil. Infancia y aprendizaje, 14(55), 87-102.

Rodríguez Arrocho, Wanda C. (2011). Aprendizaje, desarrollo y evaluación en contextos escolares: consideraciones teóricas y prácticas desde el enfoque histórico cultural. Actualidades Investigativas en Educación, 11(1), 1-36. Recuperado de http://revista.inie.ucr.ac.cr/index.php/aie/article/view/505

Rodríguez Arrocho, Wanda C. (2014). Problematización de la zona de desarrollo próximo: una tarea pendiente en la formación en psicología y educación. En Adriana Sulle y Ricardo Bur (Eds.), Vigotsky, psicólogo (pp. 117-144). Buenos Aires: Letra viva. 
Vygotsky, Lev, S. (1978). Mind in Society. The Development of Higher Psychological Processes. Cambridge, MA: Harvard University Press.

Vygotsky, Lev, S. (2000). El desarrollo de los procesos psicológicos superiores. Barcelona: Crítica.

Zapata-Rios, Miguel. (2015). Teorías y modelos sobre el aprendizaje en entornos conectados y ubicuos. Bases para un nuevo modelo teórico a partir de una visión crítica del "conectivismo. Education in the Knowledge Society (EKS) ,16(1), 69-102. 